\begin{tabular}{|c|l|}
\hline Title & $\begin{array}{l}\text { Direct simulations of homogeneous bubble nucleation: A greement with classical nucleation theory and no local hot } \\
\text { spots }\end{array}$ \\
\hline Author(s) & Diemand, Juerg; A ngelil, Raymond; Tanaka, Ky oko K.; Tanaka, Hidekazu \\
\hline Citation & $\begin{array}{l}\text { Physical review E, 90(5), 52407 } \\
\text { https://doi.org/10.1103/PhysRevE.90.052407 }\end{array}$ \\
\hline Issue Date & 201411-21 \\
\hline Doc URL & http://hdl.handle.net/2115/57838 \\
\hline Rights & @2014 A merican Physical Society \\
\hline Type & article \\
\hline Additional Information & There are other files related to thisitem in HUSCAP. Check the above URL. \\
\hline Information & PRE9052407.pdf \\
\hline
\end{tabular}

Instructions for use 


\title{
Direct simulations of homogeneous bubble nucleation: Agreement with classical nucleation theory and no local hot spots
}

\author{
Jürg Diemand, ${ }^{1,{ }^{*}}$ Raymond Angélil,,${ }^{1}$ Kyoko K. Tanaka, ${ }^{2}$ and Hidekazu Tanaka ${ }^{2}$ \\ ${ }^{1}$ Institute for Computational Sciences, University of Zurich, 8057 Zürich, Switzerland \\ ${ }^{2}$ Institute of Low Temperature Science, Hokkaido University, Sapporo 060-0819, Japan
}

(Received 29 August 2014; published 21 November 2014)

\begin{abstract}
We present results from direct, large-scale molecular dynamics simulations of homogeneous bubble (liquidto-vapor) nucleation. The simulations contain half a billion Lennard-Jones atoms and cover up to 56 million time steps. The unprecedented size of the simulated volumes allows us to resolve the nucleation and growth of many bubbles per run in simple direct micro-canonical simulations while the ambient pressure and temperature remain almost perfectly constant. We find bubble nucleation rates which are lower than in most of the previous, smaller simulations. It is widely believed that classical nucleation theory (CNT) generally underestimates bubble nucleation rates by very large factors. However, our measured rates are within two orders of magnitude of CNT predictions; only at very low temperatures does CNT underestimate the nucleation rate significantly. Introducing a small, positive Tolman length leads to very good agreement at all temperatures, as found in our recent vapor-to-liquid nucleation simulations. The critical bubbles sizes derived with the nucleation theorem agree well with the CNT predictions at all temperatures. Local hot spots reported in the literature are not seen: Regions where a bubble nucleation event will occur are not above the average temperature, and no correlation of temperature fluctuations with subsequent bubble formation is seen.
\end{abstract}

DOI: 10.1103/PhysRevE.90.052407

PACS number(s): 64.60.Q-, 05.70.Fh, 64.70.fh, 83.10.Tv

\section{INTRODUCTION}

Bubble nucleation happens in boiling and cavitation processes in a wide range of contexts and disciplines [1], e.g., the electroweak [2,3] and QCD [4] phase transitions in cosmology, direct dark matter detection experiments [5-7], vulcanism [8] and hydrodynamic cavitation erosion [9], and sonochemistry [10]. Despite its fundamental importance, the detailed mechanism of bubble nucleation remains unclear [11], and accurate predictions of bubble nucleation rates are still not yet possible [11,12].

In pure liquids, vapor bubbles must form via homogeneous nucleation, which is often suppressed by a large free energy barrier. For this reason it is possible to heat up pure liquids to a superheated, metastable state before stable bubbles form (boiling). Similarly, reducing the pressure below the saturation pressure leads to a metastable, stretched liquid and eventually to its rupture (cavitation) $[1,9,13]$.

The most widely used model to predict bubble nucleation rates is the classical nucleation theory (CNT) [9,13-16]. More recently density functional theory (DFT) $[12,17]$, square gradient theory [18] and some modifications of the classical theory $[19,20]$ have been employed to model the bubble nucleation process. Measuring bubble nucleation rates in a perfectly homogeneous liquid is very challenging in laboratory experiments [21], but can be achieved in principle in computer simulations, both with the Monte Carlo method [22] and with molecular dynamics (MD) [11,23-33]. One main conclusion of most of the MD simulations was that CNT generally underestimates bubble nucleation rates by very large factors. However, most of the existing simulations use only around 10000 or fewer atoms and could be affected by their small simulation volumes [30,31,34] and by errors from

\footnotetext{
*diemand@physik.uzh.ch; http://www.physik.uzh.ch/diemand/
}

applying methods like forward flux sampling (FFS) to the bubble nucleation process (see Ref. [35] for a discussion of possible problems). Up to now, most molecular dynamics simulations were only able to accommodate one bubble nucleation event [36], and with a large number of simulations they can constrain the mean first passage times (MFPT) [37]. However, due to unknown initial lag times and the early transient nucleation phase, the relation between MFPT and the true steady-state nucleation rate is more complex than usually assumed [38], and steady-state nucleation rate estimates based on MFPT can disagree by several orders of magnitude [39]. Here we present the first direct MD simulations which are large enough to resolve several bubble nucleation events in the steady-state nucleation phase and allow direct measurements of the bubble nucleation rates for the first time.

Section II provides a summary of the CNT, Sec. III describes our MD simulations and analysis methods. In Secs. IV, V, VI, and VII we present the results for the nucleation rates, critical sizes, size distributions, and our investigation into local hot spots preceding bubble formation. Finally, Sec. VIII concludes the paper by summarizing our findings.

\section{CLASSICAL NUCLEATION THEORY}

Classical nucleation theory (CNT) [9,13-16] estimates the work required to form a spherical vapor bubble of radius $r$ under the assumption of mechanical equilibrium,

$$
\Delta G(r)=4 \pi r^{2} \gamma-\frac{4 \pi}{3} r^{3}\left(P_{\mathrm{eq}}-P_{\mathrm{L}}\right) \delta,
$$

where $\gamma$ refers to the planar surface tension, and $P_{\mathrm{L}}$ is the ambient pressure in the liquid. The vapor pressure in the bubble $P_{\mathrm{V}}$ is expected to be slightly smaller than the equilibrium vapor pressure at saturation $P_{\mathrm{eq}}$, and the reduced pressure difference 
TABLE I. Simulation properties: initial temperature $T$, number of atoms $N$, periodic cube size $L$, atom number density $n$, and total run time.

\begin{tabular}{|c|c|c|c|c|c|}
\hline Run ID & $\begin{array}{c}T \\
{[\epsilon / k]}\end{array}$ & $N$ & $\begin{array}{c}L \\
{[\sigma]}\end{array}$ & $\begin{array}{c}n \\
{\left[\sigma^{-3}\right]}\end{array}$ & $\begin{array}{l}t_{\text {end }} \\
{[\tau]}\end{array}$ \\
\hline $\mathrm{T} 85 \mathrm{r} 1$ & 0.855 & 536870912 & 987.0 & 0.558365 & 562.5 \\
\hline $\mathrm{T} 85 \mathrm{r} 2$ & 0.855 & 536870912 & 984.0 & 0.563488 & 750.0 \\
\hline T85r3 & 0.855 & 536870912 & 981.0 & 0.568673 & 2885.0 \\
\hline $\mathrm{T} 85 \mathrm{r} 4$ & 0.855 & 536870912 & 978.0 & 0.573923 & 3250.0 \\
\hline $\mathrm{T} 85 \mathrm{r} 2 \mathrm{~h}$ & 0.855 & 67108864 & 492.0 & 0.563488 & 1500.0 \\
\hline T85r2q & 0.855 & 8388608 & 246.0 & 0.563488 & 4162.5 \\
\hline $\mathrm{T} 8 \mathrm{r} 1$ & 0.80 & 536870912 & 957.0 & 0.612537 & 300.0 \\
\hline $\mathrm{T} 8 \mathrm{r} 2$ & 0.80 & 536870912 & 954.0 & 0.618336 & 437.5 \\
\hline T8r3 & 0.80 & 536870912 & 951.0 & 0.624207 & 2375.0 \\
\hline $\mathrm{T} 7 \mathrm{r} 1$ & 0.70 & 536870912 & 921.0 & 0.687212 & 1375.0 \\
\hline $\mathrm{T} 7 \mathrm{r} 2$ & 0.70 & 536870912 & 920.5 & 0.688333 & 1300.0 \\
\hline $\mathrm{T} 7 \mathrm{r} 3$ & 0.70 & 536870912 & 919.5 & 0.690581 & 667.5 \\
\hline $\mathrm{T} 7 \mathrm{r} 4$ & 0.70 & 536870912 & 918.0 & 0.693972 & 1212.5 \\
\hline T6r1 & 0.60 & 536870912 & 898.0 & 0.741380 & 300.0 \\
\hline T6r2 & 0.60 & 536870912 & 897.0 & 0.743862 & 328.0 \\
\hline T6r3 & 0.60 & 536870912 & 896.0 & 0.746356 & 600.0 \\
\hline
\end{tabular}

is approximated with the Poynting correction factor $\delta$ :

$$
\Delta P=P_{\mathrm{V}}-P_{\mathrm{L}} \simeq\left(P_{\mathrm{eq}}-P_{\mathrm{L}}\right) \delta,
$$

and $\delta$ is given by [14]

$$
\delta \simeq 1-\left(\frac{\rho_{\mathrm{V}}}{\rho_{\mathrm{L}}}\right)+\frac{1}{2}\left(\frac{\rho_{\mathrm{V}}}{\rho_{\mathrm{L}}}\right)^{2} .
$$

A common assumption is that the pressure in the bubble equals the equilibrium vapor pressure $P_{\text {eq }}$, i.e., the Poynting correction is neglected $(\delta=1)$. Far below the critical temperature, the effect of the Poynting correction becomes very small (see Table I). In this paper we consider both cases: values assuming $\delta=1$ will be labeled CNT, and those with $\delta<1$ from Eq. (3) will be called PCNT.

The free energy $\Delta G(r)$ has a maximum at the critical radius $r_{c}$,

$$
r_{c}=\frac{2 \gamma}{\Delta P} \simeq \frac{2 \gamma}{\left(P_{\mathrm{eq}}-P_{\mathrm{L}}\right) \delta},
$$

and the height of the free energy barrier equals

$$
\Delta G\left(r_{c}\right)=\frac{16 \pi}{3} \frac{\gamma^{3}}{(\Delta P)^{2}}=\frac{4 \pi r_{c}^{2} \gamma}{3}=-\frac{4 \pi / 3 r_{c}^{3} \Delta P}{2},
$$

which is simply one third of the surface term from Eq. (1) at $r_{c}$ or minus one half of the volume term at $r_{c}$.

Small bubbles $\left(r<r_{c}\right)$ are understood to form as density fluctuations in the liquid; they are short-lived and are found at any time at a number density of

$$
n(r)=n_{\mathrm{L}} \exp \left[-\frac{\Delta G(r)}{k T}\right],
$$

where $n_{\mathrm{L}}$ is the number density in the liquid phase.

The steady-state homogeneous nucleation rate $J$ is proportional to the abundance of critical bubbles times a kinetic factor $[9,14]$ :

$$
J=n_{\mathrm{L}}\left[\frac{2 \gamma}{\pi m}\right]^{1 / 2} \exp \left[-\frac{\Delta G\left(r_{c}\right)}{k T}\right] .
$$

The prefactors in Eqs. (6) and (7) have no rigorous justification, and a range of other expressions are sometimes used. The different prefactors only differ by factors of a few, which is small compared to the large uncertainties in the exponential factor (see Ref. [40] for an overview).

\section{SIMULATION AND ANALYSIS METHODS}

\section{A. Simulation code, setup, and parameters}

The simulations were performed with the Large-scale Atomic/Molecular Massively Parallel Simulator (LAMMPS) code [41]. We use a truncated force-shifted Lennard-Jones (TSF-LJ) potential

$$
u_{\mathrm{TSF}}(r)=u_{\mathrm{LJ}}(r)-u_{\mathrm{LJ}}\left(r_{\mathrm{cut}}\right)-\left(r-r_{\mathrm{cut}}\right) \frac{d u_{\mathrm{LJ}}\left(r_{\mathrm{cut}}\right)}{d r},
$$

for $r \leqslant r_{\text {cut }}$ and $u_{\mathrm{TSF}}(r)=0$ for $r>r_{\text {cut }} . u_{\mathrm{LJ}}(r)$ is the widely used 12-6 Lennard-Jones (LJ) potential

$$
\frac{u_{\mathrm{LJ}}(r)}{4 \epsilon}=\left(\frac{\sigma}{r}\right)^{12}-\left(\frac{\sigma}{r}\right)^{6}
$$

At the cutoff radius $r_{\text {cut }}=2.5 \sigma$, both the truncated forceshifted potential $u_{\mathrm{TSF}}$ and its first derivative vanish continuously. This interaction potential is the same as used in two recent studies of bubble nucleation based on smaller simulations with a few thousand atoms $[11,31]$. The properties of the TSF-LJ fluid are given in Table II. Our simulations contain $N=2^{28}=536870912$ particles.

Four sets of simulations were performed, one to probe the boiling regime at superheated conditions at $T=0.855 \epsilon / \mathrm{k}$ (same as in Refs. [11,31]), two in the cavitation regime at $T=0.7 \epsilon / k$ (the same as in Ref. [31]) and at $T=0.6 \epsilon / k$, where negative pressures are required to trigger bubble nucleation, and one in between at $T=0.80 \epsilon / k$. The critical temperature of the TSF-LJ potential with $r_{\text {cut }}=2.5 \sigma$ is $T_{c}=0.935 \epsilon / k[11,42]$. For typical simple fluids, the onset of homogenous superheated boiling requires roughly $T>$ $0.9 \epsilon / k[9]$. Argon system units can be defined by matching the critical temperature: for the full standard LJ potential

TABLE II. Equilibrium properties of the TFS-LJ fluid: Pressure $P_{\text {eq }}$, densities of vapor and liquid phase, $\rho_{\mathrm{v}}$ and $\rho_{\mathrm{l}}$, Poynting correction $\delta$, and planar surface tension $\gamma$. Values in parentheses are $1 \sigma$ errors in the last given digit(s). These values were obtained from MD simulations of a liquid slab in equilibrium with the vapor phase by calculating the Kirkwood-Buff pressure tensor across the two planar vapor-liquid interfaces; see Refs. [44,51] for details.

\begin{tabular}{lccccl}
\hline \hline$T$ & $P_{\mathrm{eq}}$ & $\rho_{\mathrm{V}}$ & $\rho_{\mathrm{l}}$ & & \multicolumn{1}{r}{} \\
$\epsilon / k$ & {$\left[\epsilon / \sigma^{3}\right]$} & {$\left[m / \sigma^{3}\right]$} & {$\left[m / \sigma^{3}\right]$} & $\delta$ & $\begin{array}{c}\gamma \\
{\left[\epsilon / \sigma^{2}\right]}\end{array}$ \\
\hline 0.855 & $0.04610(8)$ & 0.0833 & 0.595 & 0.870 & $0.0895(24)$ \\
0.8 & $0.03028(6)$ & 0.0505 & 0.652 & 0.926 & $0.168(3)$ \\
0.7 & $0.01186(7)$ & 0.0198 & 0.729 & 0.973 & $0.329(4)$ \\
0.6 & $0.00337(4)$ & 0.00606 & 0.792 & 0.992 & $0.511(5)$ \\
\hline \hline
\end{tabular}




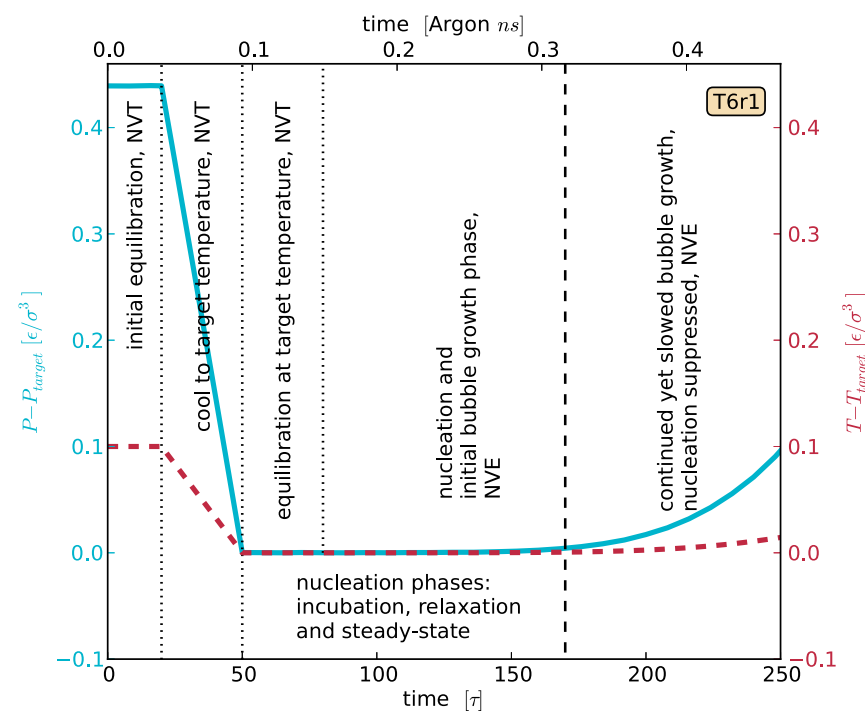

FIG. 1. (Color online) The temperature (dashed line) and pressure (solid line) differences from the targets for the setup and measurement phase of our shortest simulation, T6r1. In most runs the measurement phase is far longer. In this case, the rapid growth of only a few bubbles quickly leads to a significant pressure increase. Despite this, we are able to measure accurate nucleation rates, shown in the upper panel of Fig. 6. Size distributions and formation rates discussed in this paper are taken only during the simulations' "measurement" phase, after the setup, yet before the pressure increase.

$\epsilon / k=119.8 \mathrm{~K}$, and for the TSF-LJ potential used here one gets $\epsilon / k=161.3 \mathrm{~K}, \sigma=3.405 \AA$, $m=6.634 \times 10^{-23} \mathrm{~g}$, and $\tau=\sigma \sqrt{m / \epsilon}=1.86 \mathrm{ps}$, when converting to SI units.

The simulation box is a fixed cubic volume with periodic boundaries. We use the standard velocity-Verlet (also known as leap-frog) integrator, and the time steps are set to $\Delta t=$ $0.0025 \tau \simeq 4.65 \mathrm{fs}$. The liquid was first equilibrated in a stable state at a fixed temperature (e.g., $T=0.95 \epsilon / k$ ) for 10000 time steps. Then the temperature was reduced linearly to the target temperature of (e.g., $T=0.855 \epsilon / k$ ) over 15000 time steps and kept fixed at this temperature for another 10000 steps. During this entire setup phase the average temperature in the entire simulation box was controlled by simply rescaling all the particle velocities at every time step. After this setup period the velocity rescaling was turned of,f and the runs were continued as microcanonical simulations, i.e., with a constant number of particles, constant volume, and constant total energy $(N V E)$ : We simply and directly integrate the classical equation of motion of the atoms. No artificial constraints like thermostats or barostats are used during our simulations, and we are able to avoid applying such methods developed for systems near thermodynamic equilibrium to the highly nonequilibrium process of bubble nucleation. Figure 1 illustrates the pressure and temperature evolution over the simulation setup phase.

Energy is conserved very accurately over long time scales: in our longest run (T85r4), the total energy decreased over 1.3 million time steps, yet only by $1.7 \times 10^{-7}$ times the initial energy. For our low temperature T6 and T7 runs we use larger time steps of $\Delta t=0.004 \tau$. The total energy is still conserved within less than $2.6 \times 10^{-5}$ times the initial energy in all these runs. While no large, stable bubbles nucleate the

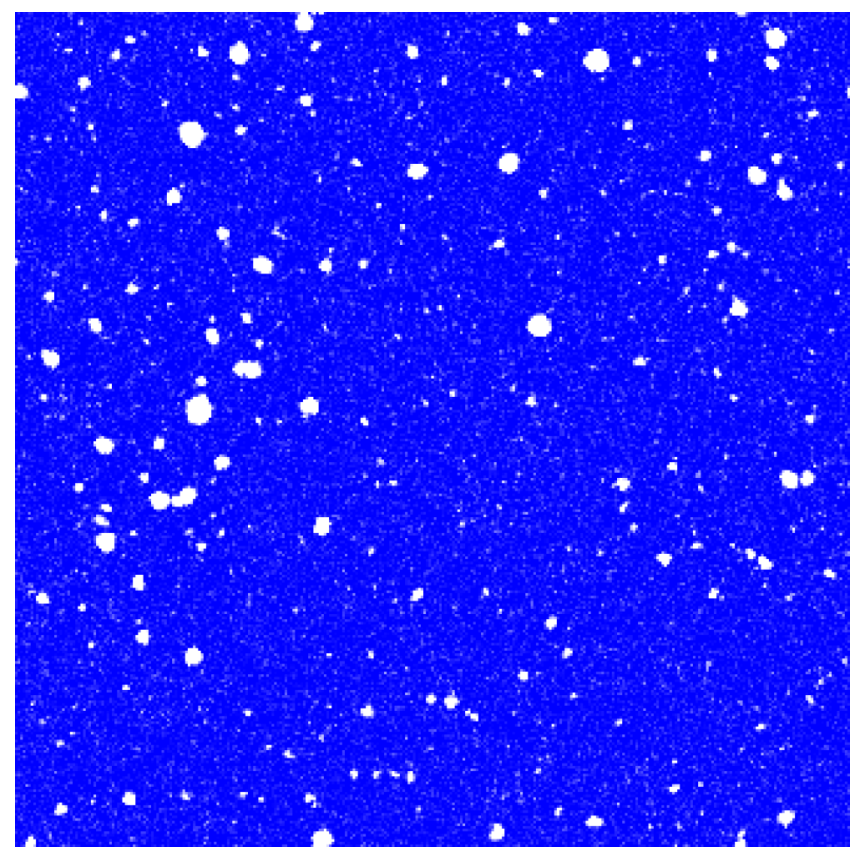

FIG. 2. (Color online) Projection through run T8r1 at $t=230 \tau$. Every pixel corresponds to a column of 319 cells of size $(3 \sigma)^{3}$. A larger number of cells below the threshold density of $0.2 \mathrm{~m} / \mathrm{\sigma}^{3}$ results in a lighter color (gray scale). This run contains about 120 large, stable bubbles at this time; see Fig. 4. See Supplemental Material for animations of such projections [46].

thermodynamic states of our systems remain constant: at the end of run T85r4 the average temperature is $T=0.85510 \epsilon / k$ and the average pressure increased by only $0.22 \%$.

\section{B. Bubble nucleation and growth}

When bubbles nucleate and surpass the critical size, they quickly reach a linear growth regime, where their radius grows at a constant, rather high speed: $v \simeq 0.03 \sigma / \tau$ at $T=0.855 \epsilon / k$ and even faster, $v \simeq 0.3 \sigma / \tau$, at $T=0.7 \epsilon / k$. The quickly growing bubbles will at some point start to occupy a significant fraction of the total simulation volume, and the pressure in the simulation box eventually increases. However, our simulation volumes are large enough to allow the formation of many critical size bubbles without a significant pressure increase in most simulations. Due to the relatively large sizes of critical bubbles and the fast growth of bubbles above the critical size, direct NVE simulation of several bubble nucleation events at constant pressure requires very large simulations and is just becoming feasible with the particle numbers we use here. For the nucleation rate analysis we only use the simulation period, where the total pressure remains within a few percent of the initial pressure. The tolerance could be set to less than $4 \%$ for most runs, but for runs $\mathrm{T} 85 \mathrm{r} 1$ and $\mathrm{T} 7 \mathrm{r} 2$ it needed to be increased to $10 \%$. to get a long enough period containing several bubble nucleation events. The end of this period is indicated by vertical dashed lines in Figure 1 and Figs. 3 to 6, and the average temperatures and pressures during the analysis period are given in Table III.

Using a large number of smaller NVE simulations (with up to 11.7 million particles) it is possible to measure the 

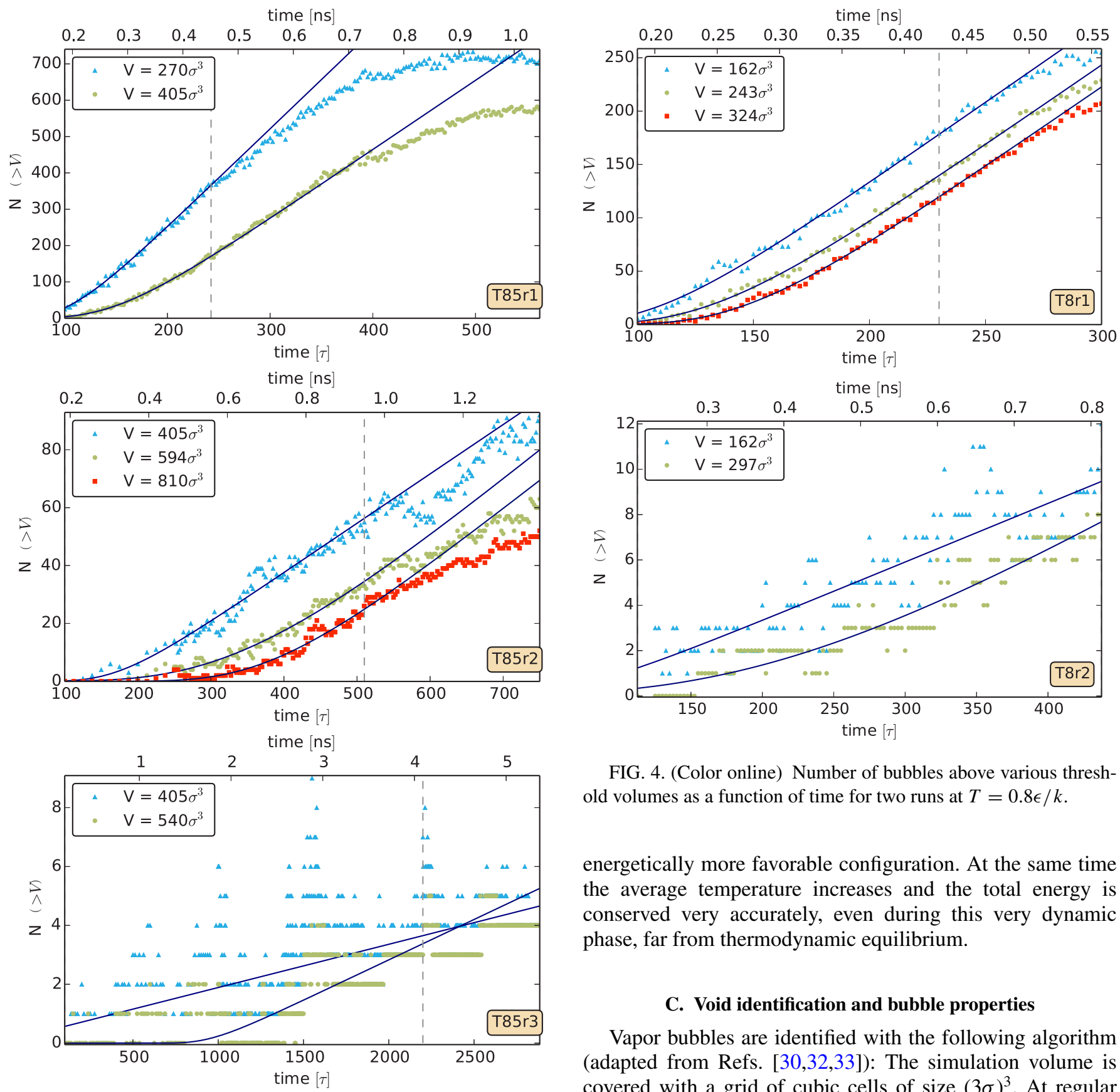

FIG. 4. (Color online) Number of bubbles above various threshold volumes as a function of time for two runs at $T=0.8 \epsilon / \mathrm{k}$.

energetically more favorable configuration. At the same time the average temperature increases and the total energy is conserved very accurately, even during this very dynamic phase, far from thermodynamic equilibrium.

\section{Void identification and bubble properties}

Vapor bubbles are identified with the following algorithm (adapted from Refs. [30,32,33]): The simulation volume is covered with a grid of cubic cells of size $(3 \sigma)^{3}$. At regular intervals (usually every 1000 time steps) the positions of all cells, which have a number density smaller than $0.2 \sigma^{-3}$ [i.e., less than six atoms within $(3 \sigma)^{3}$ ], are written out (see Fig. 2). Since $\rho_{V} \ll \rho_{L}$ at all temperatures simulated here, the identification of vapor bubbles is simple and robust. It works well for a range of threshold densities between $\rho_{V}$ and $\rho_{L}$ : We confirmed that using other density thresholds $\left(0.15 \sigma^{-3}\right.$ and $0.3 \sigma^{-3}$ ) does not affect the resulting nucleation rates, which was found also in Ref. [30]. Using different cell sizes $\left((2 \sigma)^{3}\right.$ and $\left.(4 \sigma)^{3}\right)$ does not affect the measured rates either.

Then low-density cells are linked together into individual bubbles by iteratively checking for nearby low-density cells at the 26 neighboring positions in the cubic grid. The resulting bubble volumes are stored and used for the nucleation rate measurements. We also analyzed run $\mathrm{T} 855 \mathrm{r} 2 \mathrm{~h}$ using smaller cells $(2 \sigma)^{3}$ and found the same nucleation rate. 

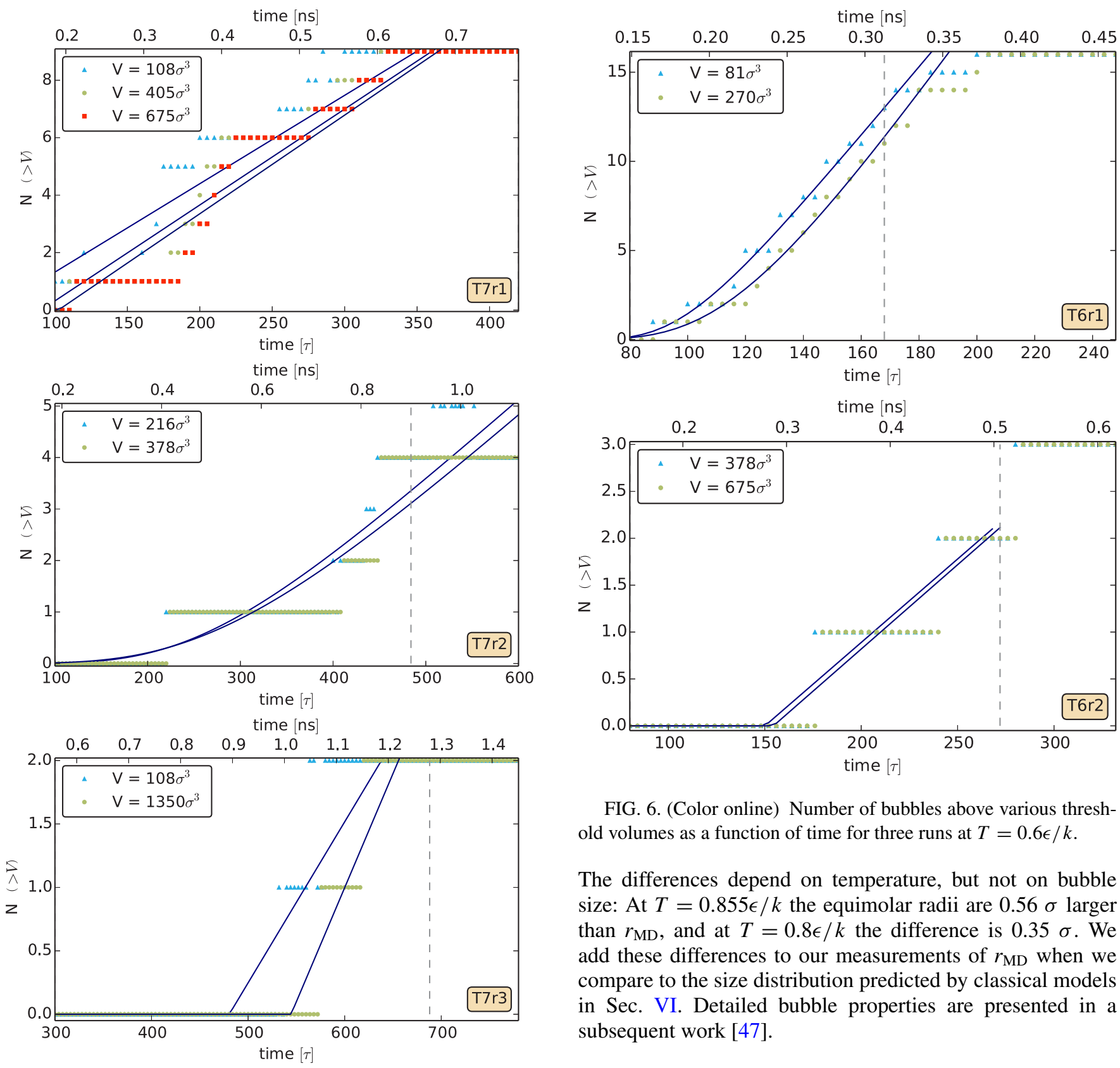

FIG. 5. (Color online) Number of bubbles above various threshold volumes as a function of time for three runs at $T=0.7 \epsilon / k$.

For a more detailed analysis of bubble properties some full simulation snapshots are written out. Bubbles near and below the critical size have significantly nonspherical, complicated shapes (as described in Ref. [31]), while the larger bubbles are roughly spherical. The vapor-liquid transitions are smooth and wide, as found in DFT [17] and square gradient [18] calculations. Comparisons with bubble density profiles show that our volume estimates $V$ based on the number of lowdensity cells are reliable: The resulting spherical radii $r_{\mathrm{MD}}=$ $(3 \mathrm{~V} / 4 \pi)^{1 / 3}$ lie only slightly below the equimolar radii, which would be the radius of a constant density bubble with a sharp interface (where the density jumps from the central value $\rho_{V}$ to the bulk liquid value $\rho_{L}$ ) and the same integrated mass [13].

FIG. 6. (Color online) Number of bubbles above various threshold volumes as a function of time for three runs at $T=0.6 \epsilon / k$.

The differences depend on temperature, but not on bubble size: At $T=0.855 \epsilon / k$ the equimolar radii are $0.56 \sigma$ larger than $r_{\mathrm{MD}}$, and at $T=0.8 \epsilon / k$ the difference is $0.35 \sigma$. We add these differences to our measurements of $r_{\mathrm{MD}}$ when we compare to the size distribution predicted by classical models in Sec. VI. Detailed bubble properties are presented in a subsequent work [47].

\section{Nucleation rate measurements}

Our large-scale direct nucleation simulations resolve several nucleation events during the steady-state nucleation regime at a nearly constant thermodynamic state. This allows us to determine the nucleation rates $J$ accurately with the Yasuoka-Matsumoto method [43,48] (also referred to as the "threshold method"), where $J$ is simply given by the slope of a linear fit to the number of bubbles $N(>V, t)$ larger than some threshold volume $V$.

Here we use a nonlinear function for $N(>V, t)$ which takes into account the time dependence of the nucleation rate before the steady state regime is reached $[13,15,49,50]$. It takes some time after the simulation setup until the first bubble nucleation event occurs (called "incubation time" in Ref. [50]) and even longer to reach the steady-state nucleation regime (related to a "relaxation time"; see Ref. [50]). For large times $\left(t>t_{0}\right)$ the number of stable bubbles grows linearly: 
$N(>V, t)=J_{\mathrm{MD}}\left(t-t_{0}\right) L^{3}$. We find that the function from Ref. [50] fits our measured $N(>V, t)$ curves very well, and that the transition time scales (i.e., incubation and relaxation time) are quite long and strongly dependent on liquid temperature and pressure (see Figs. 3 to 6).

We use a least-squares fitting algorithm and minimise the absolute differences in the bubble counts above threshold. The good statistics of some runs (T8r1 and T85r2 for example) allow a nucleation rate measurement with a relative uncertainty of around 10\%, and the results are independent of the exact value we choose for the threshold volume $V$. Most runs produce fewer stable bubble and therefore have larger uncertainties in $J_{\mathrm{MD}}$ (see Table III). Run T8r3 only produced a single stable bubble during the constant pressure epoch, which leads to a large uncertainty in $J_{\mathrm{MD}}$, while runs T85r4 and T7r4 did not nucleate at all and only allowed us to set upper limits on $J_{\mathrm{MD}}$ (see Ref. [43] for details).

In general, using larger values for the threshold volumes leads to longer lag times before the steady-state regime is reached. We therefore use the smaller of the threshold values plotted in Figs. 3 to 6 to derive the nucleation rate estimates given in Table III.

Small simulations are only able to resolve one bubble nucleation event, because the critical bubble volume is comparable to the available simulation volume. With a large number of small runs one can measure the mean first passage time accurately [37]. But due to the unknown lag time it is not possible to convert mean first passage times into accurate steady-state nucleation rates [30,39]. For the case of water vapor-to-liquid nucleation it was recently shown [39], that the mean first passage time method underestimates the true steady-state nucleation rates by about two orders of magnitude (see also Ref. [38]).

\section{E. Convergence tests}

To assess the impact of our chosen simulation box sizes on the measured nucleation rates we performed two additional simulations with the same physical properties as run T85r2, but using 8 and 64 times smaller simulation volumes. Run $\mathrm{T} 85 \mathrm{r} 2 \mathrm{~h}$ is just large enough to allow a relatively accurate nucleation rate estimate: the total pressure in the box stays within $4.5 \%$ of the initial value until $t=385 \tau$, and during that epoch we measure and average temperatures and pressures of $T=0.08512 \epsilon / k$ and $P=0.01703 \epsilon / \sigma^{3}$; we estimate a nucleation rate of $J_{\mathrm{MD}}=2.6 \pm 0.4 \times 10^{-10}$.

In the full size run (T85r2) the pressure remains within $3.5 \%$ of the target pressure up to $t=510 \tau$. The larger sample of stable bubbles forming in that period results in a tighter constraint on the steady-state nucleation rate of $J_{\mathrm{MD}}=1.8 \pm 0.2 \times 10^{-10}$. The agreement in $J$ between runs $\mathrm{T} 85 \mathrm{r} 2$ and $\mathrm{T} 85 \mathrm{r} 2 \mathrm{~h}$ within 1.5 times the standard deviation of the rate from $\mathrm{T} 85 \mathrm{r} 2 \mathrm{~h}$ is quite good, especially when the slightly higher average temperature and therefore larger superheating and higher expected nucleation rate in run $\mathrm{T} 85 \mathrm{r} 2 \mathrm{~h}$ is taken into account. This shows that our simulations with $N=536870912$ are large enough and that our nucleation rate estimates have converged. They are not affected by the finite size effects reported by Meadley and Escobedo [31] when comparing forward flux sampling (FFS) MD simulations with $N=3375$ (the size used in Wang et al. [11]) and $N=8000$.

Run T85r2q with about 8 million atoms on the other hand is too small to allow a reliable estimate of the steady-state nucleation rate. The liquid pressure does increase significantly shortly after the first bubble nucleation event. Reaching the steady-state regime and resolving several bubble nucleation

TABLE III. Average liquid temperature $T$ and pressure $P$ during the $N V E$ integration (up to the final pressure increase), pressure difference $\Delta P$ (equilibrium pressure $P_{\text {eq }}$ minus measured pressure $P$ ), critical bubble radius $r^{*}$, and nucleation rate $J$ for each run. Our critical sizes $r_{\mathrm{c}}^{*}$ were derived form the measured rates $J_{\mathrm{MD}}$ using the nucleation theorem, Eq. (12). The $r_{\mathrm{c}}^{*}$ estimates from W08 [11] and ME12 [31] are based on the FFS method. The nucleation rates were derived using CNT and PCNT and measured directly in the MD simulations. The error bars on the model predictions come from the uncertainties in the surface tensions (see Table II).

\begin{tabular}{|c|c|c|c|c|c|c|c|c|c|}
\hline Run ID & $\begin{array}{c}T \\
{[\epsilon / k]}\end{array}$ & $\begin{array}{c}P \\
{\left[\epsilon / \sigma^{3}\right]}\end{array}$ & $\begin{array}{c}\Delta P \\
{\left[\epsilon / \sigma^{3}\right]}\end{array}$ & $\begin{array}{l}r_{\mathrm{c}}^{*} \\
{[\sigma]}\end{array}$ & $\begin{array}{c}r_{\mathrm{CNT}}^{*} \\
{[\sigma]}\end{array}$ & $\begin{array}{c}r_{\mathrm{PCNT}}^{*} \\
{[\sigma]}\end{array}$ & $\begin{array}{c}J_{\mathrm{MD}} \\
{\left[\sigma^{-3} \tau^{-1}\right]}\end{array}$ & $\begin{array}{c}J_{\mathrm{CNT}} \\
{\left[\sigma^{-3} \tau^{-1}\right]}\end{array}$ & $\begin{array}{c}J_{\mathrm{PCNT}} \\
{\left[\sigma^{-3} \tau^{-1}\right]}\end{array}$ \\
\hline T85r1 & 0.8557 & 0.01438 & 0.03172 & $6.3 \pm 0.2$ & 5.64 & 6.49 & $2.8 \pm 0.3 \times 10^{-9}$ & $1.2 \times 10^{-7 \pm 0.5}$ & $1.4 \times 10^{-9 \pm 0.6}$ \\
\hline $\mathrm{T} 85 \mathrm{r} 2$ & 0.8553 & 0.01701 & 0.02909 & $6.6 \pm 0.1$ & 6.15 & 7.07 & $1.8 \pm 0.2 \times 10^{-10}$ & $8.8 \times 10^{-9 \pm 0.6}$ & $4.2 \times 10^{-11 \pm 0.8}$ \\
\hline $\mathrm{T} 85 \mathrm{r} 3$ & 0.8555 & 0.02004 & 0.02606 & $6.9 \pm 0.2$ & 6.87 & 7.90 & $2.9 \pm 0.6 \times 10^{-12}$ & $1.5 \times 10^{-10 \pm 0.7}$ & $1.9 \times 10^{-13 \pm 1.0}$ \\
\hline $\mathrm{T} 85 \mathrm{r} 4$ & 0.8551 & 0.02383 & 0.02227 & $>5.1$ & 8.04 & 9.24 & $<3.8 \times 10^{-13}$ & $7.1 \times 10^{-14 \pm 1.0}$ & $7.8 \times 10^{-18 \pm 1.3}$ \\
\hline W08 & 0.8550 & 0.02600 & 0.0201 & 6.1 & 8.91 & 10.2 & $0.9 \times 10^{-14 \pm 1}$ & $1.1 \times 10^{-16 \pm 1.2}$ & $1.5 \times 10^{-21 \pm 1.6}$ \\
\hline ME12a & 0.8550 & 0.02600 & 0.0201 & $6.4(3)$ & 8.91 & 10.2 & $2.6 \times 10^{-14 \pm 1}$ & $1.1 \times 10^{-16 \pm 1.2}$ & $1.5 \times 10^{-21 \pm 1.6}$ \\
\hline ME12b & 0.8550 & 0.02600 & 0.0201 & $7.2(5)$ & 8.91 & 10.2 & $5.1 \times 10^{-16 \pm 1}$ & $1.1 \times 10^{-16 \pm 1.2}$ & $1.5 \times 10^{-21 \pm 1.6}$ \\
\hline T8r1 & 0.8004 & -0.04379 & 0.07407 & $4.9 \pm 0.4$ & 4.54 & 4.90 & $1.7 \pm 0.3 \times 10^{-9}$ & $2.9 \times 10^{-9 \pm 0.4}$ & $1.4 \times 10^{-10 \pm 0.5}$ \\
\hline $\mathrm{T} 8 \mathrm{r} 2$ & 0.8001 & -0.03713 & 0.06741 & $5.2 \pm 0.3$ & 4.98 & 5.39 & $4.2 \pm 2.8 \times 10^{-11}$ & $6.9 \times 10^{-11 \pm 0.5}$ & $1.8 \times 10^{-12 \pm 0.6}$ \\
\hline $\mathrm{T} 8 \mathrm{r} 3$ & 0.8004 & -0.03202 & 0.06230 & $5.2 \pm 1.0$ & 5.39 & 5.83 & $1.5-22 \times 10^{-13}$ & $1.6 \times 10^{-12 \pm 0.6}$ & $2.3 \times 10^{-14 \pm 0.7}$ \\
\hline $\mathrm{T} 7 \mathrm{r} 1$ & 0.7040 & -0.16627 & 0.17813 & $3.1 \pm 1.0$ & 3.69 & 3.80 & $4.3 \pm 0.5 \times 10^{-11}$ & $7.2 \times 10^{-13 \pm 0.4}$ & $1.6 \times 10^{-13 \pm 0.4}$ \\
\hline $\mathrm{T} 7 \mathrm{r} 2$ & 0.7032 & -0.16222 & 0.17408 & $3.0 \pm 1.0$ & 3.78 & 3.88 & $2.0 \pm 1.1 \times 10^{-11}$ & $2.0 \times 10^{-13 \pm 0.4}$ & $4.2 \times 10^{-14 \pm 0.5}$ \\
\hline $\mathrm{T} 7 \mathrm{r} 3$ & 0.7002 & -0.15808 & 0.16996 & $2.8 \pm 0.8$ & 3.87 & 3.98 & $1.6 \pm 1.0 \times 10^{-11}$ & $5.1 \times 10^{-14 \pm 0.5}$ & $9.8 \times 10^{-15 \pm 0.5}$ \\
\hline $\mathrm{T} 7 \mathrm{r} 4$ & 0.7000 & -0.14724 & 0.15910 & $>3.0$ & 4.14 & 4.25 & $<1.6 \times 10^{-12}$ & $7.9 \times 10^{-16 \pm 0.5}$ & $1.2 \times 10^{-16 \pm 0.6}$ \\
\hline ME12 & 0.7000 & -0.15000 & 0.16186 & 3.1 & 4.06 & 4.18 & $3.9 \times 10^{-11 \pm 1}$ & $2.5 \times 10^{-15 \pm 0.5}$ & $4.0 \times 10^{-16 \pm 0.5}$ \\
\hline T6r1 & 0.6090 & -0.34779 & 0.35116 & $3.1 \pm 0.4$ & 2.91 & 2.93 & $2.9 \pm 1.0 \times 10^{-10}$ & $3.4 \times 10^{-14 \pm 0.4}$ & $2.1 \times 10^{-14 \pm 0.4}$ \\
\hline T6r2 & 0.6005 & -0.33596 & 0.33933 & $2.9 \pm 0.4$ & 3.01 & 3.03 & $2.5 \pm 1.2 \times 10^{-11}$ & $4.0 \times 10^{-15 \pm 0.4}$ & $2.4 \times 10^{-15 \pm 0.4}$ \\
\hline T6r3 & 0.6025 & -0.32375 & 0.32712 & $2.9_{-2.9}^{+0.6}$ & 3.12 & 3.15 & $0.9-14 \times 10^{-12}$ & $3.4 \times 10^{-16 \pm 0.4}$ & $2.0 \times 10^{-16 \pm 0.4}$ \\
\hline
\end{tabular}


events at roughly constant ambient pressure is not possible with direct $(N V E)$ simulations of this size.

\section{F. Equilibrium simulations}

Comparing the results of our bubble nucleation simulations with theoretical models requires knowledge of the thermodynamic properties of the TFS-LJ fluid, especially the vapor and liquid densities at saturation $\left(\rho_{\mathrm{v}}\right.$ and $\left.\rho_{1}\right)$, the pressure at coexistence $P_{\text {eq }}$, and the planar surface tension $\gamma$. Some of these values can be found in the literature [11], but the statistical uncertainties in $\gamma$ are too large for our purposes.

To estimate these quantities we performed a series of equilibrium MD simulations of liquid-vapor systems. At each temperature one relatively large system (5 million atoms) consisting of a liquid slab with vapor on both sides was set up and equilibrated for a large number of time steps (typically one million) at fixed temperature $(N V T)$ and with periodic boundaries (see Refs. [44,51] for details).

When the system has reached a perfectly stable equilibrium state it is evolved for another one million NVT time steps. At every time step the Kirkwood-Buff pressure tensor across the two planar vapor-liquid interfaces is calculated, and the time-averaged values stored every 50000 steps, and converted into estimates of $\rho_{\mathrm{v}}, \rho_{\mathrm{l}}, P_{\mathrm{eq}}$, and $\gamma$. This is done 20 times to determine the average values and the $1 \sigma$ scatter for these quantities. The large simulation size and long time integration leads to less statistical uncertainty compared to earlier smaller and shorter simulations. We can then constrain the planar surface tension $\gamma$ within a relative one sigma error of $2.6 \%$ or less, while earlier estimates have larger uncertainties of around $10 \%$. However, even the remaining small uncertainties in the surface tension lead to uncertainties of around one order of magnitude in the theoretical nucleation rate predictions (see Fig. 7 and Table III).

At $T=0.855 \epsilon / k$ we can compare our values to earlier estimate for the same TFS-LJ fluid at the same temperature: Wang et al. [11] found $\gamma=0.098 \pm 0.008$, which agrees well with our estimate within their large statistical uncertainty. The estimate from density functional theory (DFT) $\gamma=0.119$ [52], however, seems too high. Both estimates are higher than ours and therefore lead to significantly lower model predictions for the nucleation rate. The far better agreement with the classical models we find here is caused not only to our lower measured rates, but also by our lower surface tension estimates, which increase the theoretical nucleation rate predictions by large factors.

\section{NUCLEATION RATES}

Figures 3 to 6 show the number of bubbles larger than some threshold volume as a function of time. Using the threshold method, these counts are used to derive nucleation rate estimates as described in Sec. IIID. The steady-state bubble nucleation rates obtained from our direct $N V E$ MD simulations are given in Table III, and in the next two sections we compare them to predictions from classical models and to earlier estimates from smaller simulations.

\section{A. Comparison with classical models}

It is widely believed that classical models generally underestimate bubble nucleation rates by very large factors $[11,12,24,26,31]$ and that they perform significantly worse than in the case of vapor-to-liquid nucleation [12]. However, we do find very good agreement with the classical theory (both with CNT and PCNT) at high temperatures $(T=0.8 \epsilon / k$ and $0.855 \epsilon / k$ ). The classical nucleation rate predictions match quite well in the superheated boiling regime $(T=0.8 \epsilon / k)$ and also for moderate cavitation cases (slightly negative pressures and $0.8 \epsilon / k$ ); see Fig. 7 .

At lower temperatures the measured nucleation rates are significantly higher than the CNT predictions, and the ratio $J / J_{\mathrm{CNT}}$ reaches values around $10^{4}$ at $T=0.6 \epsilon / \mathrm{k}$. As in the case of vapor-to-liquid condensation, CNT seems to predict realistic rates at some intermediate or high temperature and to underestimate the rates below that temperature [43]. And the predicted temperature dependence is off by a similar amount: decreasing the temperature by $10 \%$ increases the ratio $J / J_{\mathrm{CNT}}$ by about one order of magnitude in both the bubble nucleation and the vapor-to-liquid case [43] in the nucleation rate regime accessible to large-scale MD simulations.

\section{B. Implications for the size dependence of the surface tension}

The large ratios of measured-to-predicted nucleation rates $J / J_{\mathrm{PCNT}}$ at low temperatures may indicate that the surface tension depends on bubble size. Bubbles near the critical scale determine the nucleation rates, and this scale is smaller at low temperatures. A suitable correction to the planar surface tension [40,53], which results in a lower surface tension for small bubbles would lead to a smaller energy barrier and higher nucleation rates at low temperatures.

Assuming that the classical models are correct except for the assumed size independent surface tension, one can use the nucleation rates from the simulations for an indirect estimate of the surface tension of critical size bubbles [40]. Using the reconstructed free energy landscapes (Figs. 10 and 11) such estimates can in principle be made for all sizes represented in the simulated size distribution [45].

The correction introduced by Tolman [54] is

$$
\gamma_{T}(r)=\gamma /\left(1+2 \delta_{T} / r\right) \simeq \gamma\left(1-2 \delta_{T} / r\right) .
$$

A small, positive Tolman length of $\delta_{T}=0.25 \sigma$ would lead to perfect agreement at low temperatures $\left(J / J_{\mathrm{PCNT}} \simeq 1\right.$ at $T=0.6 \epsilon / k$ and $0.7 \epsilon / k)$, and it would not spoil the good agreement between the MD simulations and the classical models at the higher temperatures $\left(J / J_{\mathrm{PCNT}} \simeq 0.1\right.$ at $T=$ $0.8 \epsilon / k$ and $0.855 \epsilon / k$ ); see Fig. 7. This agrees well with the Tolman length of $\delta_{T}=0.5 r_{0} \simeq 0.32 \sigma$ derived from largescale vapor-to-liquid nucleation simulations [45], using the somewhat different standard LJ potential with $r_{\text {cut }}=5 \sigma$. Our estimate of $\delta_{T}=0.25 \sigma$ is also consistent with the constraint $\left|\delta_{T}\right| \leqslant 0.5 \sigma$ obtained by Horsch et al. [55] for equilibrium TFS-LJ droplets, with the practically constant $\gamma$ found for equilibrium LJ bubbles with $r>6 \sigma$ by Matsumoto and Tanaka [56], but not with the stronger size dependence reported in Akbarzadeh et al. [57]. Note, however, that estimating the surface tension of small equilibrium MD nanobubbles is extremely challenging [58]. 

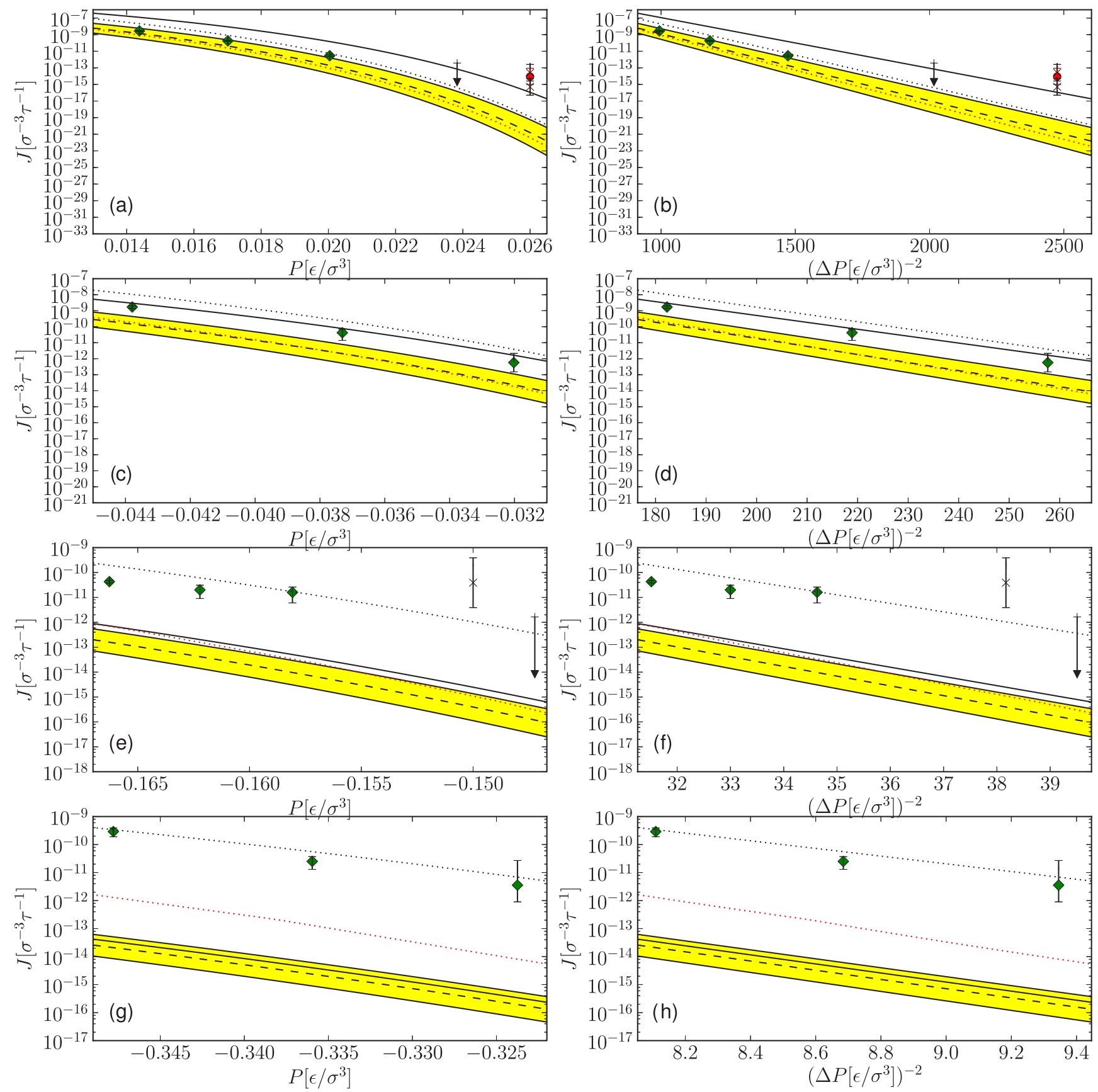

FIG. 7. (Color online) Measured nucleation rates at $k T / \epsilon=0.855$ (a, b), 0.8 (c, d), 0.7 (e, f), and 0.6 (g, h) against ambient pressure (left column) and against the inverse of the pressure difference squared (right column). Our MD results (diamonds, down arrows for upper limits) are compared with the CNT (solid lines) and PCNT (dashed lines) predictions and to the MD FFS simulation results from Wang et al. [11] (circles) and Meadley and Escobedo [31] (crosses). The shaded areas around the PCNT model predictions illustrate the effect of the $1 \sigma$ uncertainty in our planar surface tension estimates. The dotted lines are PCNT-like predictions with bubble size-dependent surface tensions: black (upper) dotted lines show the Tolman correction with $\delta_{T}=0.25 \sigma$, red (lower) dotted lines use the surface tension from Eq. (11).

By cutting out spherical voids of various sizes in molecular dynamics simulations of a liquid under negative pressure, and observing whether the resultant bubble is stable, Ref. [28] estimates a Tolman length $\delta_{T}=0.26 \pm 0.01 \sigma$ at $k T=0.6$. This is very close to our estimate of $\delta_{T}=0.25 \epsilon$ at $k T=0.6 \epsilon$.

Baidakov and Bobrov [40] use a different approximation, which is applicable to a wider range of bubble sizes,

$$
\gamma_{T}(r)=\gamma /\left(1+2 \delta_{B} / r+l^{2} / r^{2}\right)
$$

and includes a second parameter $l$. With similar parameters as in Ref. [40] $\left(\delta_{B}=-0.1 \sigma\right.$ and $\left.l=1.0 \sigma^{2}\right)$ the predicted nucleation rates at low temperatures are still too low by two to three orders of magnitude. The correction is too small at the critical sizes of 3 to $4 \sigma$, which are relevant for the nucleation rates in our low temperature runs (see Fig. 8). The correction becomes large at the smaller critical scales of 1 to $2 \sigma$ relevant in the runs in Badiakov and Bobrov [40] and may be specific to the regime of nucleation 


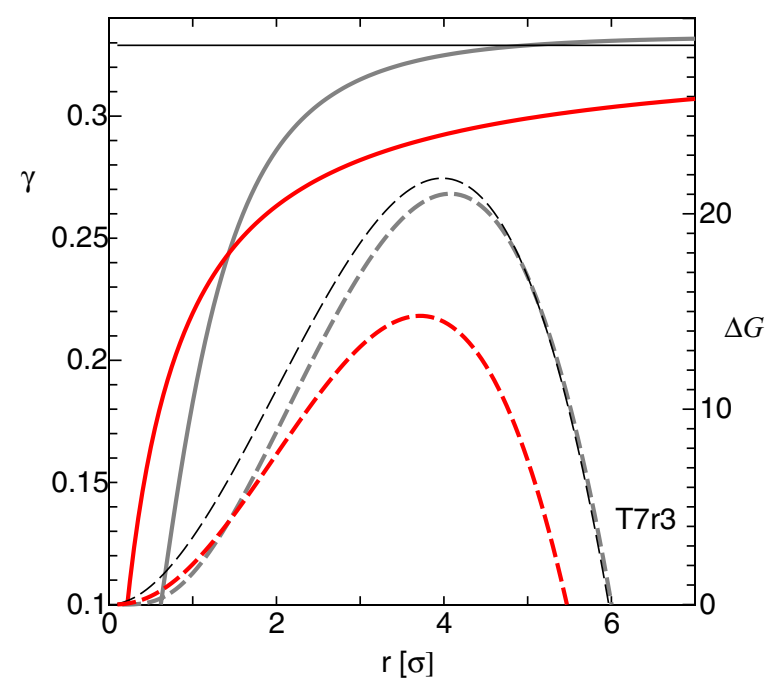

FIG. 8. (Color online) Surface tensions (solid lines) as a function of bubble radius at $T=0.7 \epsilon / k$ : The planar surface tension (thin solid), a small, positive Tolman length of $\delta_{T}=0.25 \sigma$ (red solid line, lower one at large radii) and using Eq. (11) with $\delta_{B}=-0.1 \sigma$ and $l=1.0 \sigma^{2}$ (gray solid line, upper one at large radii). Dashed lines show the corresponding free energy curves for run $\mathrm{T} 7 \mathrm{r} 3$ (see axis label on the right): PCNT assumes the constant planar surface tension (thin). The other two $\Delta G$ curves use a small, positive Tolman length (red) and Eq. (11) (gray).

rates, temperatures, and interparticle potential probed in their simulations.

These corrections likely depend on temperature. A small, positive Tolman length of $\delta_{T}=0.25 \sigma$ fits the nucleation rates well at all temperatures, but it would lead to worse fits to the free energy landscapes reconstructed from the bubble size distribution at higher temperatures (Figs. 10 and 11). A sizeindependent surface tension (as found in Ref. [56]) actually matches the results from our high-temperature runs better.

\section{Comparisons with other simulations}

Figure 9 shows the measured nucleation rates divided by the PCNT estimate as a function of temperature relative to the critical temperature. The relative temperature scale makes comparisons between results from simulations which use different intermolecular potentials possible: Our direct MD results and the MD FFS simulation results from Wang et al. [11] and Meadley and Escobedo [31] employed a TFS-LJ intramolecular potential with a cutoff scale of $r_{\text {cut }}=2.5 \sigma$, which results in a fluid with $T_{c}=0.935$. The other studies used standard LJ potential without a force shift and with cutoff scale of from $r_{\text {cut }}=2.5 \sigma$ to $r_{\text {cut }}=6.578 \sigma$, which gives rise to critical temperatures from $T_{c}=1.19$ to $T_{c}=1.31$ [59].

For the comparison we calculated $J / J_{\mathrm{CNT}}$ using the both the $J$ and the $J_{\mathrm{PCNT}}$ (or $J_{\mathrm{CNT}}$, the differences are very small at low temperature) values given in each paper. Only for Refs. [11,31], which simulated the exact same TFS-LJ at the same temperatures as this work, do we recalculate $J_{\mathrm{PCNT}}$. Note the uncertainties in the $J_{\mathrm{CNT}}$ and $J_{\mathrm{PCNT}}$ are quite large, mostly due to the uncertainties in the planar surface tension estimates $\gamma$. Figure 9 does not show these errors. For the case of our

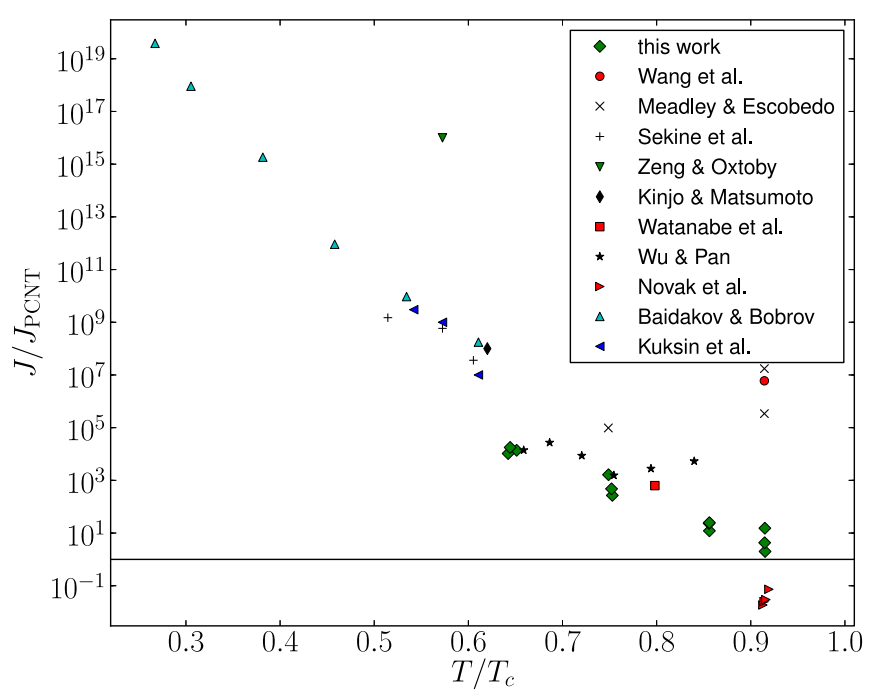

FIG. 9. (Color online) Measured nucleation rates divided by the PCNT estimate against $T / T_{c}$ for our simulations (green diamonds) and bubble nucleation results from the literature.

$J_{\text {PCNT }}$ estimates, they are illustrated by the shaded regions in Fig. 7.

The majority of bubble nucleation studies find significantly larger nucleation rates than predicted by CNT and PCNT, i.e., $J / J_{\mathrm{CNT}}$ of $10^{5}$ and larger. This seems to be in qualitative agreement with the density functional calculation by Zeng and Oxtoby [12], which predict a very large $J / J_{\mathrm{CNT}} \sim 10^{16}$. Our simulations offer an way to cross-check these earlier results, because they are quite different from all the previous simulations and in many aspects more accurate and reliable: We use simple and robust direct $N V E$ MD simulations which are more than a factor of 1000 times larger than all previous bubble nucleation simulations (except for the spinodal nucleation simulations of Ref. [30], which contain up to $10^{7}$ atoms). Our runs are the first which are demonstrably not affected by finite size effects. We find very good agreement with PCNT and only a relatively small increase in $J / J_{\mathrm{CNT}}$ as we go to lower temperatures. We do not find the large $J / J_{\mathrm{CNT}}$ values found in most earlier studies. Our results agree well with the relatively small deviations from classical models found by Wu and Pan [24], Watanabe et al. [30], and Novak et al. [25].

Some of the differences between the simulation results shown in Fig. 9 may be due to the different intermolecular potentials used. For example the standard LJ potential with $r_{\text {cut }}=6.578 \sigma$ used in Baidakov and Bobrov [40] has a far larger planar surface tension. Even after rescaling with the corresponding critical temperature $T_{c}$, the differences remain large: at $0.76 T_{c}$ for example, the $\gamma$ value of the force-shifted $r_{\text {cut }}=2.5 \sigma$ potential we use here is about a factor of two smaller. A larger surface tension leads to smaller critical bubble sizes, it is therefore plausible that the numerical experiments in Baidakov and Bobrov [40] and similar studies probe a different regime, where bubble curvature and atomistic effects play a larger role and where classical models are therefore less accurate. Their size-dependent fit to the bubble surface tension actually leads to good fits to our nucleation rates (see Sec. IV B), their larger $J / J_{\mathrm{CNT}}$ values may therefore be due 
to the smaller critical bubbles in the regime probed in their simulations.

Other studies [11,31] do use the exact same potential at very similar thermodynamic states, and can be compared directly with our results: Both find significantly larger $J / J_{\mathrm{PCNT}}$ factors than we do (see also Fig. 7). Meadley and Escobedo [31] show that their results are affected by the size of the simulations, their nucleation rates decrease when 8000 instead of 3375 atoms are used. $J$ was likely overestimated due to the small simulations sizes used in these studies [31] and perhaps also due to artifacts in the FFS method [35].

\section{CRITICAL SIZES FROM THE FIRST NUCLEATION THEOREM}

The first nucleation theorem can be used to calculate the volume of critical bubbles from the nucleation rates directly $[13,60,61]$. It does not rely on a specific model (like CNT for example) and provides a useful independent estimate of the critical bubble size. Wilemski [61] showed that the critical volume is

$$
V_{c}=\frac{\rho_{\mathrm{L}}}{\rho_{\mathrm{L}}-\rho_{\mathrm{V}}}\left(\frac{\partial \ln J}{\partial P_{\mathrm{L}}}\right)_{T} .
$$

We estimate the derivative by taking the finite differences to the next available nucleation rate at the same temperature. If both a higher and a lower rate are available, these two rates are used to calculate the slope. Uncertainties in the nucleation rate estimates $J_{\mathrm{MD}}$ are propagated into an error estimate on the critical sizes by using the shallowest and steepest slopes allowed by the $1 \sigma$ errors on $J_{\mathrm{MD}}$. The resulting estimates of $V_{c}$ are converted into a spherical radius $r_{c}$ and listed in Table III.

We find that the critical bubble sizes from the nucleation theorem are in very good agreement with the predictions from both classical models, CNT and PCNT at all temperatures. While the predicted nucleation rates agree with the simulations only at the higher temperatures, the pressure dependence (i.e., the slopes in the left-hand panels of Fig. 7) of the nucleation rates seems to be predicted accurately by the classical models at all temperatures. Similar conclusions have been reached in the case of vapor-to-liquid nucleation in Refs. [43,62-64].

\section{FREE ENERGY FOR BUBBLE FORMATION}

If the free energy as a function of bubble size $\Delta G(r)$ is known, one can calculate the equilibrium bubble size distribution directly with Eq. (6). During steady-state nucleation, this distribution is only realized for small bubble sizes $\left(r \ll r_{c}\right)$. The steady-state distribution turns out to be a factor of two lower at $r_{c}$ and flat for larger bubble sizes [15]. Conversely, one can measure the steady-state size distribution and the nucleation rate in a large scale MD simulation and use them to reconstruct the entire free energy function $\Delta G(r)$, even at $r_{c}$ and above [45].

Figures 10 and 11 show the free energy for bubble formation. The bubble size distributions were measured in the MD simulations and time-averaged over the steady-state nucleation phase at constant liquid pressure. Together with our MD nucleation rate measurement [and assuming that the empirical pre-exponential factor in Eq. (7) is correct] this

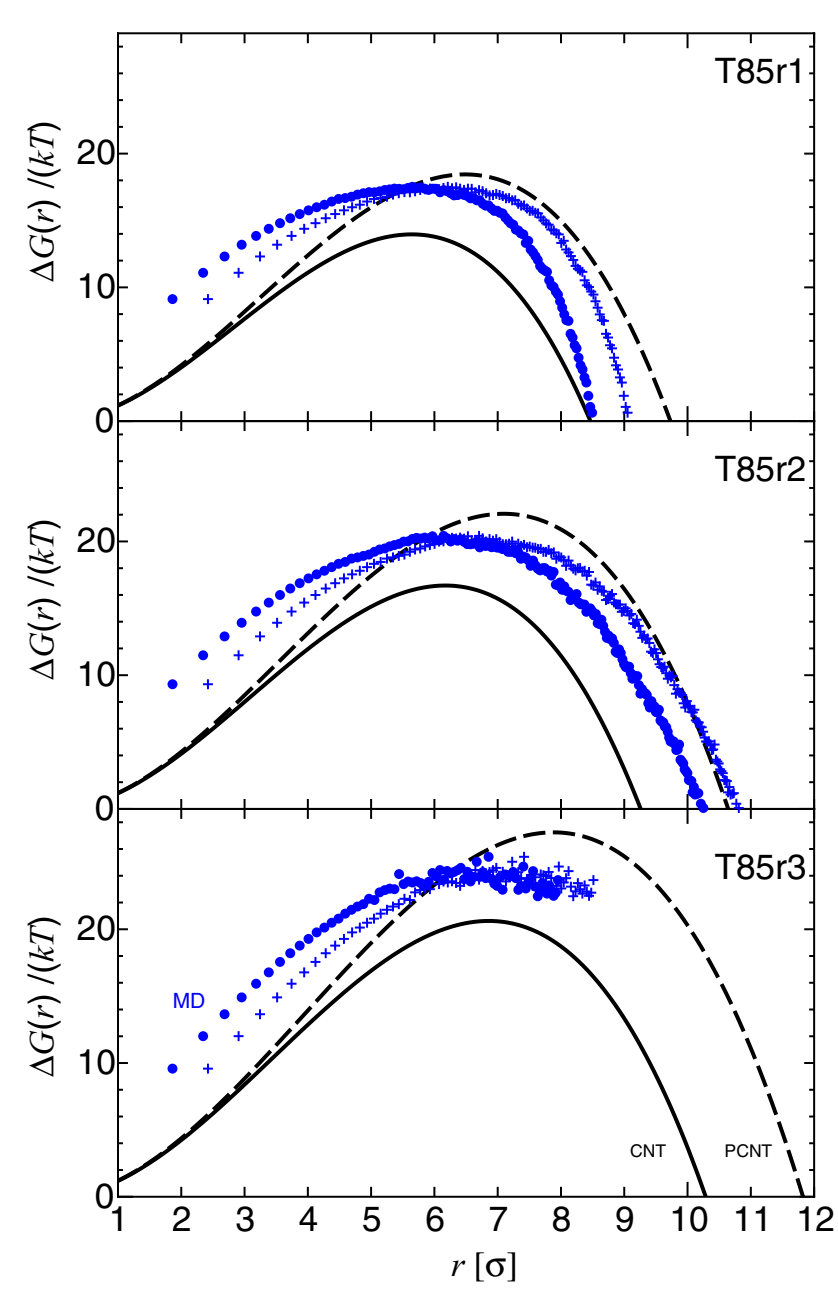

FIG. 10. (Color online) Free energy for bubble formation at $k T / \epsilon=0.855$. Reconstructed free energy curve from the MD bubble size distribution in terms of $r_{\mathrm{MD}}$ (circles) and in terms of the estimated equimolar radius $\left(r_{\mathrm{MD}}+0.56 \sigma\right)$ (crosses). Model predictions are shown with solid (CNT) and dashed (PCNT) lines.

allows the reconstruction of the full free energy function with the method from Tanaka et al. [45]. The bubble sizes were estimated by converting the volume of all cells below the threshold density of $0.2 \mathrm{~m} / \sigma^{3}$ into a spherical radius $r_{\mathrm{MD}}$. Comparing these radii with the equimolar radii of a wide range of large, stable bubble reveals that our $r_{\mathrm{MD}}$ values are slightly smaller, by an offset which depends on temperature, but not on bubble size. In Figs. 10 and 11 we use both $r_{\mathrm{MD}}$ as well as the estimated equimolar radii.

The reconstructed free energy landscape agrees well with CNT and PCNT at these high temperatures. At the lower temperature the bubbles are too small to allow a detailed comparison with this method. The peak position $\left(r_{c}\right)$ agrees with PCNT at both temperatures. The peak height matches the PCNT prediction perfectly at $k T / \epsilon=0.855$ and is slightly lower than PCNT at $k T / \epsilon=0.8$. This is consistent with the excellent agreement of the nucleation rates at $k T / \epsilon=0.855$, and the slightly larger $J_{\mathrm{MD}}$ versus $J_{\mathrm{PCNT}}$ at $k T / \epsilon=0.8$ (Fig. 7).

The models assume mechanical equilibrium at all sizes, as well as a sharp density transition from vapor to liquid, while our simulated bubbles have transition regions which are 


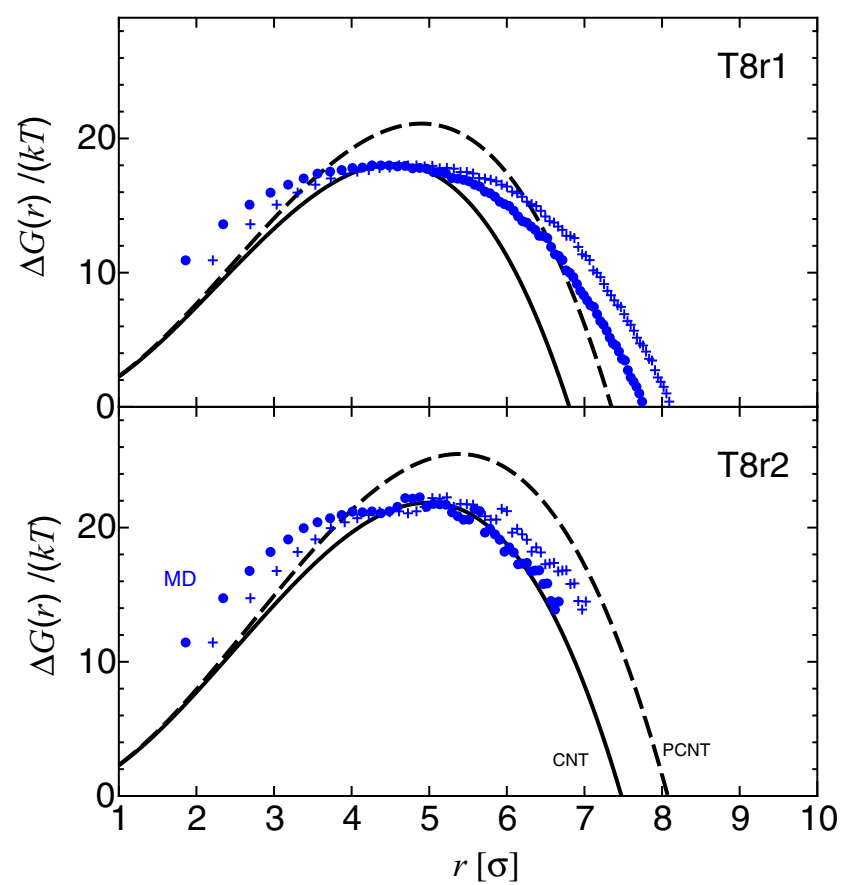

FIG. 11. (Color online) Free energy for bubble formation at $k T / \epsilon=0.8$. Reconstructed free energy curve from the MD bubble size distribution in terms of $r_{\mathrm{MD}}$ (circles) and in terms of the estimated equimolar radius $\left(r_{\mathrm{MD}}+0.35 \sigma\right)$ (crosses). Model predictions are shown with solid (CNT) and dashed (PCNT) lines.

several $\sigma$ wide [47], and their exact radii are difficult to define. For comparisons with classical models one often uses the equimolar radius. Indeed, the curves shifted to larger radii to approximate the equimolar radii match the predictions slightly better. The agreement is surprisingly good, given the many differences between the model assumptions and the actual properties of our MD bubbles (wide interfaces, nonspherical shapes, nonisothermal effects, etc. [47]).

\section{LOCAL HOT SPOTS}

In a recent study Wang et al. [11] reported that in their MD simulations the occurrence of local temperature fluctuations ("hot spots") correlates strongly with subsequent bubble formation. This process is not present in the CNT and may explain the far larger nucleation rates found in their MD simulations in comparison to the CNT prediction. However, at the same temperature as used in Wang et al. $(T=0.855 \epsilon / k)$ we find far lower rates and relatively good agreement with CNT (see Sec. IV). In this section we show that there are no local hot spots preceding bubble nucleation in our simulations. There only is a small amount of extra kinetic energy in bubble-forming locations, which is perfectly consistent with the amount of movement required to make room for the bubbles within the liquid.

Local kinetic energies and densities were measured for 200000 time steps during the steady-state nucleation regime in run $\mathrm{T} 85 \mathrm{r} 2 \mathrm{hs}$. Both quantities were measured in cells of size $(3 \sigma)^{3}$ at every step, time-averaged over 500 steps and then stored on disk. Cells with a density below 0.2 are identified as bubble-forming cells, and the first time the density falls below

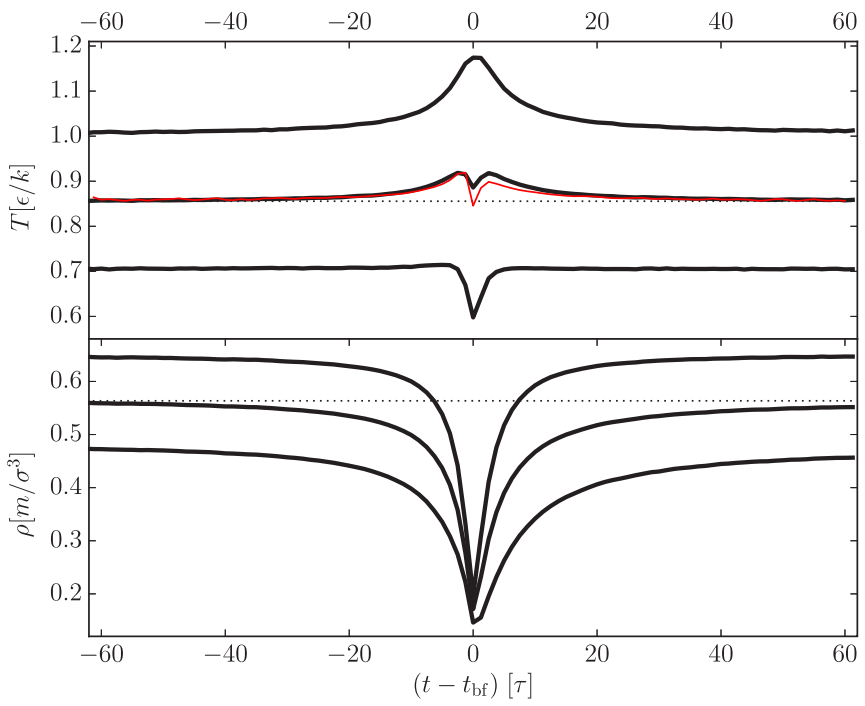

FIG. 12. (Color online) Average local temperature (top panel) and density (bottom panel) in bubble-forming cells of size $(3 \sigma)^{3}$ as a function of time since the moment of bubble formation $t_{\mathrm{bf}}$ (thick solid lines). The upper and lower thick solid lines indicate the one sigma cell-to-cell scatter, dotted lines the averages over the entire simulation volume. The estimated effect of extra kinetic energy from growing and shrinking bubbles is shown with a red thin solid line; see text for details.

the threshold is stored as the moment of bubble formation $t_{b f}$. In this way 234000 bubble-forming cells were found, corresponding to $5.3 \%$ of the 4.4 million cells which cover the entire simulation volume. For these cells we averaged the local kinetic energies and densities at the same times relative to their moment of bubble formation $t_{b f}$ and plot these averages as a function of $\left(t-t_{b f}\right)$; see Fig. 12 .

Near the moment of bubble formation $t_{\mathrm{bf}}$, the average kinetic energy in bubble-forming cells is indeed slightly higher than in the entire simulation volume. The excess becomes as large as $7 \%$ and is comparable to the hot spot signal reported in Fig. 6 in Wang et al. [11]. However, the excess kinetic energy does not precede bubble formation: it appears at the same time as the averaged density drops, and it exactly matches the amount of liquid movement required to obtain these lower densities.

A simple model can match and explain this excess kinetic energy: assuming a spherical vapor bubble with a sharp interface, fully enclosed within the cubic cell. The vapor is assumed to be at the equilibrium density $\left(\rho_{v}=0.0833 \mathrm{~m} / \sigma^{3}\right)$ and at a slightly lower temperature $(T=0.80 \epsilon / k)$, both in agreement with the vapor properties we measure in large bubbles [47]. The liquid is assumed to have the same average density and temperature as the entire simulation. At each time $\left(t-t_{b f}\right)$ a bubble radius is calculated, so that the total density in the cell matches the average density measured in the simulation. Mass conservation implies by how much each radial shell of the liquid around the bubble has to move as the bubble radius changes with time. These velocities are added to the thermal motion in the radial direction, the total kinetic energy of the vapor and liquid in the cell is calculated and then 


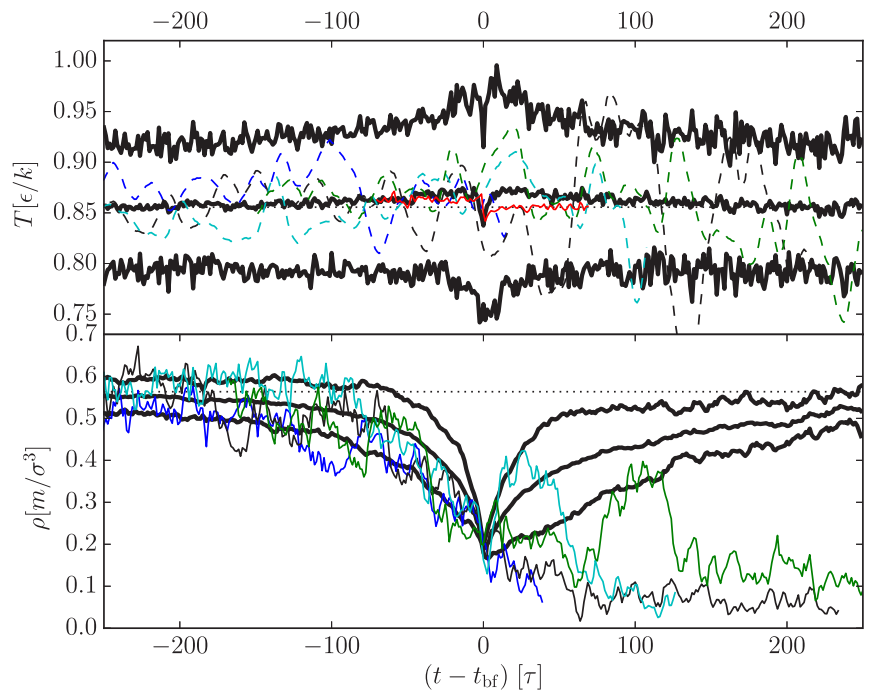

FIG. 13. (Color online) Same as Fig. 12 but using larger cells of size $(6 \sigma)^{3}$. The values in the central cells of four bubble nucleation events are given with dotted lines (top panel) and thin solid lines (bottom panel); see text for details.

converted into an average cell temperature (thin lines in the top panels of Figs. 12 and 13).

Despite several unrealistic assumptions (sharp interface, spherical shape, fully enclosed within cell), this simple model reproduces the observed excess kinetic energy very well. We conclude that bubble formation is not preceded by local hot spots. Bubbles form out of an isothermal liquid, as assumed in the CNT. The apparently higher local temperatures in bubbleforming cells near $t_{\mathrm{bf}}$ are simply caused by the inevitable rearrangement of liquid as bubbles form (and disappear), which introduces some extra kinetic energy into the liquid phase around the bubbles.

Figure 12 shows the same analysis using larger cells of size $(6 \sigma)^{3}$. Due to a smaller number of cells, the statistics are worse and this figure is noisier than Fig. 13. However, the results are the very similar: The kinetic energy in bubble-forming cells is above the simulation average, but well matched by the extra kinetic energy required to grow (or shrink) the cavities required to fit the average local density decrease (or increase). Here only 306 out of 551368 cells ever fall below the density threshold. A significant fraction of those belong to long-lived or even large, stable bubbles. Therefore the average density in these cells increases quite slowly after $t_{\mathrm{bf}}$ and remains below the simulation average even long after $t_{\mathrm{bf}}$.

At $t \simeq t_{\mathrm{bf}} \pm 2 \tau$ the average local temperatures lie below the simulation average. In large stable bubbles we measure an average vapor density of about $T=0.80 \epsilon / k$, below the simulation average of $T=0.855 \epsilon / k$ due to the latent heat used during evaporation; see Ref. [47]. Simply assuming that these cells contain a mixture of cooler vapor at $T=0.80 \epsilon / k$ and isothermal liquid at $T=0.855 \epsilon / k$, both at their equilibrium densities, matches the observed drop in the average local temperatures quite well.

Figures 12 and 13 also indicate that the one sigma cellto-cell scatter in the local densities and local kinetic energies is quite large. These are real fluctuations, not just sampling noise, since for example the local density in a $(3 \sigma)^{3}$ cell averaged over 500 measurements has only about $1 \%$ sampling error. Large local temperature fluctuations are present in the entire simulation volume, but they do not correlate at all with subsequent local bubble nucleation events. The excess in the average kinetic energy right before (and also right after) $t_{\mathrm{bf}}$ is significantly smaller than a $1 \sigma$ fluctuation, and it is not a temperature fluctuation ("local hot spot"), but simply caused by the movement required to achieve the density changes.

Four large stable bubbles form during the steady-state nucleation regime in run $\mathrm{T} 85 \mathrm{r} 2 \mathrm{~h}$. Their centers move less than $2.5 \sigma$ during the analysis period, and each center lies within one fixed $(6 \sigma)^{3}$ cell at practically all times. The local densities and kinetic energies of the central cells of these four bubbles (time-averaged over 500 measurements) are shown in Fig. 13: They show wild fluctuations, with an amplitude similar the one sigma scatter found in the other cells. The kinetic energies were smoothed using a Gaussian window with a width of $5 \tau$ to make the figure more easily viewable. The histories of these four large bubbles are obviously very noisy, and no time before $t_{\mathrm{bf}}$ can one identify a period were their formation is preceded by a local hot spot. The average temperature of these four regions of successful bubble nucleation fluctuate randomly around the simulation average at all times before the moment of bubble formation $t_{\mathrm{bf}}$.

\section{CONCLUSIONS}

The main results presented in this work can be summarized as follows:

(1) Large-scale, microcanonical (NVE) MD runs allow the simulation of homogeneous bubble nucleation in a realistic way, free of unphysical numerical manipulations (thermostating and barostating) and unaffected by finite simulation size effects.

(2) Bubble nucleation rates are lower than most of the previous estimates from smaller simulations.

(3) Our measured bubble nucleation rates agree well with classical model in the regimes of superheated boiling (positive ambient pressure) and moderate cavitation (moderately negative pressures): They lie within two orders of magnitude of CNT predictions. The reconstructed free energy landscapes also agree very well with CNT in these regimes.

(4) In the extreme cavitation regime (at very large negative pressures) $\mathrm{CNT}$ does underestimate the nucleation rates significantly.

(5) Introducing a small, positive Tolman length $\left(\delta_{T}=\right.$ $0.25 \sigma$ ) leads to very good agreement between the predicted and measured nucleation rates at all temperatures. The same conclusion was reached from our recent large scale vapor-toliquid nucleation simulations [45].

(6) The critical bubbles sizes derived with the nucleation theorem agree well with the CNT predictions at all temperatures.

(7) Local hot spots reported in an earlier MD simulation [11] are not seen: Regions where bubble nucleation events will occur are not above the average temperature, and we 
observe no correlation between temperature fluctuations and subsequent bubble formation.

Our direct large-scale bubble nucleation simulations form a large number of stable bubbles in a realistic manner and environment. They produce a lot of additional information about these bubbles, like their shapes, density and temperature profiles and growth rates. These properties will be presented in a subsequent work [47].

\section{ACKNOWLEDGMENTS}

The authors thank V. Kalikmanov for useful discussion. Computations were preformed on SuperMUC at Leibniz Supercomputing Centre, Garching, on Monte Rosa at the Swiss National Supercomputing Centre, Lugano, and on the zBox4 at University of Zurich. J.D. and R.A. are supported by the Swiss National Science Foundation.
[1] P. G. Debenedetti, Metastable Liquids: Concepts and Principles (Princeton University Press, Princeton, 1996).

[2] M. Dine, R. G. Leigh, P. Huet, A. Linde, and D. Linde, Phys. Rev. D 46, 550 (1992).

[3] A. Mégevand and A. D. Sánchez, Phys. Rev. D 77, 063519 (2008).

[4] L. S. Kisslinger, S. Walawalkar, and M. B. Johnson, Phys. Rev. D 71, 065017 (2005).

[5] M. Felizardo, T. Girard, T. Morlat, A. Fernandes, A. Ramos, J. Marques, A. Kling, J. Puibasset, M. Auguste, D. Boyer et al., Phys. Rev. Lett. 108, 201302 (2012).

[6] S. Archambault, F. Aubin, M. Auger, E. Behnke, B. Beltran, K. Clark, X. Dai, A. Davour, J. Farine, R. Faust et al., Phys. Lett. B 682, 185 (2009).

[7] A. Pullia, Adv. High Energy Phys. 2014, 387493 (2014).

[8] H. Massol and T. Koyaguchi, J. Volcanol. Geotherm. Res. 143, 69 (2005).

[9] C. E. Brennen, Cavitation and Bubble Dynamics (Cambridge University Press, Cambridge, 2013).

[10] K. S. Suslick, Science 247, 1439 (1990).

[11] Z. Wang, C. Valeriani, and D. Frenkel, J. Phys. Chem. B 113, 3776 (2008).

[12] X. C. Zeng and D. W. Oxtoby, J. Chem. Phys. 94, 4472 (1991).

[13] D. Kashchiev, Nucleation (Butterworth-Heinemann, Oxford, 2000).

[14] M. Blander and J. L. Katz, AIChE J. 21, 833 (1975).

[15] K. Kelton and A. L. Greer, Nucleation in Condensed Matter: Applications in Materials and Biology, Vol. 15 (Elsevier, Amsterdam, 2010).

[16] V. I. Kalikmanov, Nucleation Theory, Lecture Notes in Physics (Springer, Dordrecht, 2013).

[17] V. K. Shen and P. G. Debenedetti, J. Chem. Phys. 114, 4149 (2001).

[18] Ø. Wilhelmsen, D. Bedeaux, S. Kjelstrup, and D. Reguera, J. Chem. Phys. 140, 024704 (2014).

[19] C. F. Delale, J. Hruby, and F. Marsik, J. Chem. Phys. 118, 792 (2003).

[20] V. K. Shen and P. G. Debenedetti, J. Chem. Phys. 118, 768 (2003).

[21] V. E. Vinogradov, P. A. Pavlov, and V. G. Baidakov, J. Chem. Phys. 128, 234508 (2008).

[22] V. K. Shen and P. G. Debenedetti, J. Chem. Phys. 111, 3581 (1999).

[23] T. Kinjo and M. Matsumoto, Fluid Phase Equilib. 144, 343 (1998).

[24] Y. W. Wu and C. Pan, Microscale Thermophys. Eng. 7, 137 (2003).
[25] B. R. Novak, E. J. Maginn, and M. J. McCready, Phys. Rev. B 75, 085413 (2007).

[26] M. Sekine, K. Yasuoka, T. Kinjo, and M. Matsumoto, Fluid Dyn. Res. 40, 597 (2008).

[27] S. Tsuda, S. Takagi, and Y. Matsumoto, Fluid Dyn. Res. 40, 606 (2008).

[28] A. Y. Kuksin, G. E. Norman, V. V. Pisarev, V. V. Stegailov, and A. V. Yanilkin, Phys. Rev. B 82, 174101 (2010).

[29] J. L. Abascal, M. A. Gonzalez, J. L. Aragones, and C. Valeriani, J. Chem. Phys. 138, 084508 (2013).

[30] H. Watanabe, M. Suzuki, and N. Ito, Phys. Rev. E 82, 051604 (2010).

[31] S. L. Meadley and F. A. Escobedo, J. Chem. Phys. 137, 074109 (2012).

[32] H. Watanabe, M. Suzuki, and N. Ito, Comput. Phys. Commun. 184, 2775 (2013).

[33] H. Watanabe, M. Suzuki, H. Inaoka, and N. Ito, arXiv:1407.0102.

[34] A. Y. Kuksin, I. Morozov, G. Norman, V. Stegailov, and I. Valuev, Mol. Simul. 31, 1005 (2005).

[35] K. Torabi and D. S. Corti, J. Phys. Chem. B 117, 12491 (2013).

[36] An exception are the recent multibubble simulations by Watanabe et al. [32,33]. They are of similar scale as the ones presented here; however, they do probe the spinodal regime and do not measure nucleation rates.

[37] J. Wedekind, R. Strey, and D. Reguera, J. Chem. Phys. 126, 134103 (2007)

[38] V. A. Shneidman, J. Chem. Phys. 141, 051101 (2014).

[39] A. V. Mokshin and B. N. Galimzyanov, J. Chem. Phys. 140, 024104 (2014).

[40] V. G. Baidakov and K. S. Bobrov, J. Chem. Phys. 140, 184506 (2014).

[41] S. Plimpton, J. Comput. Phys. 117, 1 (1995).

[42] J. R. Errington, P. G. Debenedetti, and S. Torquato, J. Chem. Phys. 118, 2256 (2003).

[43] J. Diemand, R. Angélil, K. K. Tanaka, and H. Tanaka, J. Chem. Phys. 139, 074309 (2013).

[44] R. Angélil, J. Diemand, K. K. Tanaka, and H. Tanaka, J. Chem. Phys. 140, 074303 (2014).

[45] K. K. Tanaka, J. Diemand, R. Angélil, and H. Tanaka, J. Chem. Phys. 140, 194310 (2014).

[46] See Supplemental Material at http://link.aps.org/supplemental/ 10.1103/PhysRevE.90.052407 and http://www.ics.uzh.ch/pl2X-sim-diemand/movies for bubble formation movies. They show the evolution of the projected densities visualized in the same way as in Fig. 2. 
[47] R. Angélil, J. Diemand, K. K. Tanaka, and H. Tanaka, Phys. Rev. E (to be published).

[48] K. Yasuoka and M. Matsumoto, J. Chem. Phys. 109, 8451 (1998).

[49] V. A. Shneidman, K. A. Jackson, and K. M. Beatty, Phys. Rev. B 59, 3579 (1999).

[50] V. A. Shneidman, in Nucleation and Atmospheric Aerosols (Springer, Dordrecht, 2007), pp. 158-161.

[51] V. Baidakov, S. Protsenko, Z. Kozlova, and G. Chernykh, J. Chem. Phys. 126, 214505 (2007).

[52] J. F. Lutsko, Phys. Rev. E 74, 021603 (2006).

[53] V. I. Kalikmanov, J. Chem. Phys. 124, 124505 (2006).

[54] R. C. Tolman, J. Chem. Phys. 17, 333 (1949).

[55] M. Horsch, H. Hasse, A. K. Shchekin, A. Agarwal, S. Eckelsbach, J. Vrabec, E. A. Müller, and G. Jackson, Phys. Rev. E 85, 031605 (2012).
[56] M. Matsumoto and K. Tanaka, Fluid Dyn. Res. 40, 546 (2008).

[57] H. Akbarzadeh, H. Abroshan, F. Taherkhani, C. Izanloo, and G. A. Parsafar, Chem. Phys. 381, 44 (2011).

[58] I. A. Cosden and J. R. Lukes, J. Heat Transfer 133, 101501 (2011).

[59] A. Trokhymchuk and J. Alejandre, J. Chem. Phys. 111, 8510 (1999).

[60] I. J. Ford, Phys. Rev. E 56, 5615 (1997).

[61] G. Wilemski, J. Chem. Phys. 125, 114507 (2006).

[62] J. Wedekind, J. Wölk, D. Reguera, and R. Strey, J. Chem. Phys. 127, 154515 (2007).

[63] K. K. Tanaka, K. Kawamura, H. Tanaka, and K. Nakazawa, J. Chem. Phys. 122, 184514 (2005).

[64] K. K. Tanaka, H. Tanaka, T. Yamamoto, and K. Kawamura, J. Chem. Phys. 134, 204313 (2011). 